\title{
POSTERIOR SCLERITIS AND POLYMYALGIA RHEUMATICA
}

\author{
IAN G. SIMMONS, ERNA E. KRITZINGER and PHILIP I. MURRAY \\ Birmingham
}

\begin{abstract}
SUMMARY
Scleritis is typically a non-infectious granulomatous inflammatory process. It may be found in conjunction with systemic disease, more commonly in those patients with necrotising anterior scleritis. ${ }^{1,2}$ We describe a patient with posterior scleritis and polymyalgia rhematica. This association has not previously been reported.
\end{abstract}

\section{CASE REPORT}

A 77-year-old woman presented with a 6 week history of a painful, red right eye associated with reduced visual acuity. Twelve months previously she had been diagnosed by a consultant geriatrician as having polymyalgia rheumatica (PMR) after a 6 week history of lethargy, anorexia, weight loss, morning stiffness, pain and stiffness in the muscles of the shoulder and pelvic girdles, neck, knees and hands. Her erythrocyte sedimentation rate (ESR) was raised at $84 \mathrm{~mm} / \mathrm{h}$ and there was no evidence of other forms of systemic vasculitis, with negative rheumatoid factor, antinuclear and antineutrophil cytoplasmic antibodies and normal levels of von Willebrand factor. At that time she had been started on prednisolone $15 \mathrm{mg} /$ day which resulted in a rapid improvement in symptoms over 1 week. A superficial temporal artery biopsy had been performed which was negative for giant cell arteritis. After a few months the prednisolone was reduced to $10 \mathrm{mg} / \mathrm{day}$ then gradually down to $5 \mathrm{mg} /$ day. Her prednisolone had been increased to $15 \mathrm{mg} /$ day 2 weeks prior to being seen by an ophthalmologist because she developed eye pain, general malaise and intermittent nausea. UK.

From: Birmingham and Midland Eye Centre, Birmingham,

Correspondence to: Prof P. I. Murray, PhD, FRCS, FRCOphth, Academic Unit of Ophthalmology, Birmingham and Midland Eye Centre, City Hospital NHS Trust, Dudley Road, Birmingham B18 7QU, UK. Tel: +44 (0121) 507 6851. Fax: +44 (0121) 5075853.
On examination, her visual acuities were $6 / 60$ in her right eye and 6/9 in the left. She had an inferior exudative retinal detachment with macular pucker on the right and an annulociliary chorioretinal detachment on the left. B-scan ultrasonography showed scleral thicknesses of $3.1 \mathrm{~mm}$ right and $2.0 \mathrm{~mm}$ left and demonstrated the left annulociliary detachment. A diagnosis of bilateral posterior scleritis was made and she was treated with methylprednisolone $1 \mathrm{~g}$ intravenously and her oral prednisolone increased to $40 \mathrm{mg} /$ day. Her ESR was $45 \mathrm{~mm} / \mathrm{h}$.

Seven days later her symptoms had eased considerably and the vision improved in her right eye to $6 / 24$. The right posterior scleral thickness was now $2.6 \mathrm{~mm}$. There were bilateral shallow exudative retinal detachments and the ESR had fallen to $18 \mathrm{~mm} / \mathrm{h}$. After 2 weeks her visual acuities were 6/12 right, 6/9 left and her oral corticosteroids were reduced to $20 \mathrm{mg} /$ day.

Over the next 3 weeks her condition deteriorated. She again complained of anorexia and malaise. Although her eyes appeared white with no evidence of anterior segment disease, there had been an increase in the sub-retinal exudation with a corresponding drop in visual acuity (6/36 right, $6 / 12$ left). A fluorescein angiogram revealed delayed circulation with disc hypoperfusion in both eyes. There was an oedematous area, supero-temporal to the right fovea, which showed no early fluorescence but some mottled staining in the later films. The posterior scleral thickness had increased to $5.9 \mathrm{~mm}$ on the right (Fig. 1) and $1.8 \mathrm{~mm}$ on the left. Her oral corticosteroids were increased to $30 \mathrm{mg} /$ day.

She continued to worsen, and after a further 3 weeks presented as an emergency with marked pain and injection of both anterior sclera. Her vision was hand movements rights, $6 / 18$ left. There was a right proptosis of $3 \mathrm{~mm}$ with associated reduction in ocular movement and a right relative afferent pupillary defect. The exudative retinal detachments had

Eye (1997) 11, 727-728 C 1997 Royal College of Ophthalmologists 


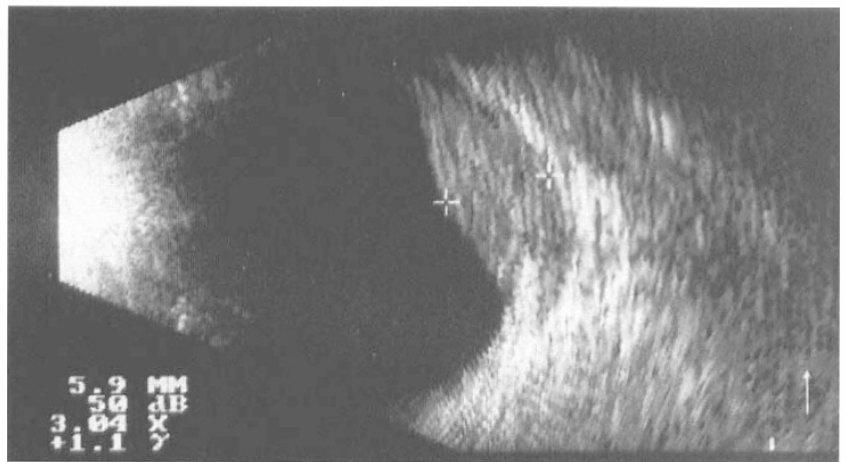

Fig. 1. B-scan ultrasound of the right eye showing a maximum posterior scleral thickness of $5.9 \mathrm{~mm}$.

increased in size. Whilst the left posterior scleral thickness remained relatively normal, the right had increased to $10 \mathrm{~mm}$. Serum immunochemistry was again normal as was a chest radiograph. Magnetic resonance imaging confirmed the scleral thickening and there was no evidence of an orbital mass.

Despite a pulse of intravenous methylprednisolone $1 \mathrm{~g}$ and commencement of methotrexate $7.5 \mathrm{mg} /$ week, the visual acuity in the right eye failed to improve. Nevertheless, her pain resolved and her visual acuities remained at hand movements right, $6 / 12$ left. Her current medication is oral prednisolone $15 \mathrm{mg} /$ day and methotrexate $15 \mathrm{mg} /$ week. The last documented measurement of the right posterior scleral thickness was $3.2 \mathrm{~mm}$. She remains a difficult on-going problem as the posterior scleritis flares up whenever her corticosteroids and/or immunosuppressives are reduced. A left posterior subcapsular cataract subsequently developed and this was successfully treated with a standard extracapsular extraction and implantation of a heparin surface modified posterior chamber intraocular lens.

\section{COMMENT}

Scleritis covers a spectrum of ocular disease that ranges from mild self-limiting episodes of inflammation to a painful, sight-threatening, destructive necrotising process. Almost $50 \%$ of patients with scleritis have a systemic vasculitic disease, which is a sign of poor general and ocular prognosis because it indicates serious systemic and ocular complications. ${ }^{3}$ The most frequent associations are with rheumatic conditions, in particular those with a vasculitic component, such as rheumatoid arthritis and Wegener's granulomatosis. Other associations include relapsing polychondritis, systemic lupus erythematosus, the spondyloarthropathies and Behçet's disease.

There are a range of protocols for the treatment of scleritis. For mild disease, oral non-steroidal antiinflammatory drugs (NSAIDs) are the initial choice. Topical steroids are usually used as supplementary therapy rather than primary treatment. Failure of the inflammation to resolve or clinical evidence of more severe disease would require the introduction of oral corticosteroids. For those patients who fail to improve or who develop scleral necrosis, oral immunosuppressive agents such as cyclophosphamide, azathioprine, cyclosporin or methotrexate are added, and pulsed immunosuppression in the form of intravenous methylprednisolone and cyclophosphamide may be necessary. Despite these medications, therapeutic failure is not uncommon and more likely in those patients just taking NSAIDs, but drug intolerance is more frequent in patients on systemic immunosuppressive therapy. ${ }^{4}$

Although anterior scleritis with PMR has been recognised, ${ }^{2}$ to our knowledge this is the first report in association with posterior scleritis. This case reemphasises that posterior scleritis is a potentially sight-threatening condition and its treatment can require the use of systemic corticosteroids and immunosuppressive agents ${ }^{3,4}$ which may need to be continued over a protracted period.

Key words: Posterior scleritis, Polymyalgia rheumatica.

\section{REFERENCES}

1. Sainz de la Maza M, Jabbur NS, Foster CS. Severity of scleritis and episcleritis. Ophthalmology 1994;101: 389-96.

2. Tuft SJ, Watson PG. Progression of scleral disease. Ophthalmology 1991;98:467-71.

3. Sainz de la Maza M, Foster CS, Jabbur NS. Scleritis associated with systemic vasculitic diseases. Ophthalmology 1995;102:687-92.

4. Sainz de la Maza M, Jabbur NS, Foster CS. An analysis of therapeutic decision for scleritis. Ophthalmology 1993;100:1372-6. 